\title{
Association between presenting motor symptoms and the risk of cognitive impairment in Parkinson's disease
}

\author{
M Viitanen, J A Mortimer, D D Webster
}

\begin{abstract}
Neuropsychological data collected from two groups of patients with idiopathic Parkinson's disease $(n=50,159)$ were analysed to investigate the association between presenting motor symptoms determined from retrospective chart review and the risk of cognitive impairment. Presenting motor symptoms were abstracted from the medical records and coded by type, location, and laterality. Longitudinal data on changes in the maximum speed of voluntary arm movements were available for a subsample of patients. Bilateral ( $v$ unilateral) presentation was associated with an increased risk of cognitive impairment an average of nine years after onset of disease as measured by memory tests and the minimental state examination. A higher rate of decline of arm movement speed was also predictive of greater memory dysfunction. The type, side (left $v$ right), and location (lower $v$ upper extremity) of the presenting symptoms were not, however, consistently associated with the risk of cognitive impairment later in the course of the disease.
\end{abstract}

(F Neurol Neurosurg Psychiatry 1994;57:1203-1207)

Education and

Clinical Center,

Veterans Affairs

Medical Center,

Minneapolis,

Minnesota, USA

$M$ Viitanen

J A Mortimer

Department of

Geriatric Medicine,

Karolinska Institutet,

Huddinge Hospital,

Sweden

$M$ Viitanen

Department of

Neurology, University

of Minnesota Medical

School, USA

J A Mortimer

D D Webster

Department of

Epidemiology,

University of

Minnesota School of

Public Health,

Minneapolis,

Minnesota, USA

J A Mortimer

Correspondence to:

Dr M Viitanen, Department

of Geriatric Medicine,

Karolinska Institutet,

Huddinge Hospital, S-141

86 Huddinge, Sweden.

Received 18 October 1993

and in revised form

30 March 1994.

Accepted 21 April 1994 Cognitive impairment is a well known symptom of Parkinson's disease, especially in elderly patients in later stages of the illness. Impairments in verbal, visuospatial, executive, and memory functions have been described. ${ }^{12}$

Several investigators have studied the association between motor and cognitive symptomatology. Cooper and coworkers ${ }^{3}$ found that cognitive function correlated weakly with motor disability in newly diagnosed, untreated, non-depressed, and nondemented idiopathic parkinsonian patients. This finding led them to conclude that the pathology of the early cognitive impairment is largely independent of the motor disorder. In another study, cognitive disorder was found to be poorly correlated with akinesia and rigidity, but to be strongly related to gait disorder and dysarthria. ${ }^{4}$ Other investigators have reported significant correlations between the severity of bradykinesia and impaired performance on tests assessing visual-spatial reasoning and psychomotor speed in patients with Parkinson's disease treated with levodopa. ${ }^{56}$
Whereas it is likely that the severity of motor dysfunction is correlated with the presence of cognitive deficits after the disease is well established, there have been no reports to date regarding the relation of the nature of the presenting motor symptoms to the risk of cognitive decline later in the disease. In the present study we examined this association in two cohorts of patients for whom detailed neuropsychological data and uniform documentation of disease presentation were available.

\section{Methods}

Neuropsychological data from two groups of patients with idiopathic Parkinson's disease were analysed. The first group comprised 60 patients identified from consecutive appointments at a large clinic ${ }^{57}$ and the second group consisted of 159 different patients from the same clinic eight years later. Patients were considered to have idiopathic Parkinson's disease if they had two cardinal features of Parkinson's disease (resting tremor, bradykinesia, rigidity, or parkinsonian gait impairment) and did not have a secondary cause for the disorder. Patients with postencephalic or drug induced parkinsonism were excluded, as were patients with progressive supranuclear palsy, presumed striatonigral degeneration, olivopontocerebellar degeneration, or essential tremor. Patients whose disease began with cognitive impairment were also excluded.

Table 1 shows the demographic character-
Table 1 Demographic data for patients studied

\begin{tabular}{lll}
\hline & Group 1 & Group 2 \\
\hline Number of patients & 60 & 159 \\
Women/men (n) & $2 / 60$ & $3 / 159$ \\
Age (y) & $62 \cdot 7(8 \cdot 2)$ & $66 \cdot 4(8 \cdot 5)^{\star}$ \\
Duration of parkinsonian & $9 \cdot 4(8 \cdot 1)$ & $9 \cdot 3(8 \cdot 1) \mathrm{NS}$ \\
$\quad$ symptoms (y) & $11 \cdot 4(2 \cdot 8)$ & $12 \cdot 0(3 \cdot 2) \mathrm{NS}$ \\
Education $(\mathrm{y})$ & &
\end{tabular}
cantly younger than group $2 \quad(t=2 \cdot 952$, $\mathrm{p}=0.004)$, but there were no other significant differences. Differences in the mean ages of parkinsonian patients in the two groups reflected the primary source of patients in the clinic-namely, veterans from the second world war and the Korean war. Over a period of eight years, the average age of new patients to the clinic increased by about four years. Almost all were men, characteristic of the veteran population from which they were 
Table 2 Factor structure for neuropsychological battery given to patients in group 1 $(n=50)$

\begin{tabular}{|c|c|c|c|}
\hline \multirow[b]{2}{*}{ Variables } & \multicolumn{3}{|l|}{ Factor } \\
\hline & $\begin{array}{l}1 \\
\text { Memory }\end{array}$ & $\begin{array}{l}2 \\
\text { Visuospatial } \\
\text { abilities }\end{array}$ & $\begin{array}{l}3 \\
\text { Psychomotor } \\
\text { Speed }\end{array}$ \\
\hline WMS logical memory, paragraph 1 , immediate recall & $0 \cdot 852$ & $0 \cdot 047$ & $0 \cdot 161$ \\
\hline WMS logical memory, paragraph $1,1 \mathrm{~h}$ delay recall & $0 \cdot 830$ & $0 \cdot 134$ & 0.095 \\
\hline WAIS vocabulary & $0 \cdot 824$ & $0 \cdot 103$ & -0.095 \\
\hline WMS logical memory, paragraph 2 , immediate recall & 0.796 & 0.275 & $0 \cdot 225$ \\
\hline WMS logical memory, paragraph $2,1 \mathrm{~h}$ delay recall & $0 \cdot 764$ & 0.317 & $0 \cdot 189$ \\
\hline WAIS Information & 0.735 & $0 \cdot 178$ & -0.201 \\
\hline WMS paired associates, hard items, $1 \mathrm{~h}$ delay recall & 0.663 & 0.304 & 0.085 \\
\hline WMS paired associates, hard items, immediate recall & $0 \cdot 610$ & 0.364 & 0.048 \\
\hline Spatial orientation memory (errors) & -0.538 & -0.596 & 0.007 \\
\hline Trails B (time) & 0.283 & -0.784 & $0 \cdot 224$ \\
\hline Trails B (errors) & $-0 \cdot 199$ & $-0 \cdot 780$ & 0.085 \\
\hline Wepman visual discrimination (errors) & -0.327 & -0.740 & -0.235 \\
\hline Trails A (time) & $-0 \cdot 149$ & -0.714 & $-0 \cdot 228$ \\
\hline WAIS block design & $0 \cdot 367$ & 0.693 & 0.004 \\
\hline Bender recall & $0 \cdot 420$ & 0.668 & $0 \cdot 139$ \\
\hline Trails A (errors) & 0.099 & -0.651 & -0.166 \\
\hline WAIS digit symbol & $0 \cdot 349$ & 0.646 & $0 \cdot 221$ \\
\hline Finger tapping rate-right & 0.021 & $0 \cdot 177$ & 0.844 \\
\hline Finger tapping rate-left & 0.084 & $0 \cdot 168$ & $0 \cdot 830$ \\
\hline Cancellation task (time) & -0.090 & $-0 \cdot 203$ & -0.654 \\
\hline Cancellation task (errors) & -0.093 & -0.489 & $0 \cdot 361$ \\
\hline WMS paired associates, easy items, $1 \mathrm{~h}$ delay recall & $0 \cdot 387$ & 0.336 & $0 \cdot 321$ \\
\hline WMS paired associates, easy items, immediate recall & $0 \cdot 332$ & 0.486 & $0 \cdot 228$ \\
\hline WAIS digit span & $0 \cdot 483$ & $0 \cdot 160$ & 0.050 \\
\hline Variance explained (\%) & $25 \cdot 7$ & $23 \cdot 3$ & $10 \cdot 7$ \\
\hline
\end{tabular}

* Rotated loading for each factor are shown in boxes.

drawn. As there was no reason to assume that the women would show different associations, data from the small number of women were analysed together with those from the men. At the time of neuropsychological testing 52 of the 60 patients in group 1 and 146 of the 159 patients in group 2 were being treated with levodopa or Sinemet. Initial motor symptoms at disease onset as described by the patients were systematically recorded by the same neurologist (DDW) at entry into the clinic. These symptoms were abstracted from the medical records and coded by type (bradykinesia/akinesia, tremor, rigidity, gait apraxia), location (lower extremity, upper extremity, lower and upper extremities, other parts of body), and laterality (left sided, right sided, bilateral, not lateralisable). Side effects when levodopa was first given were recorded as no side effects, psychotic symptoms, dyskinesia, or both. Age of onset was estimated as the age at which patients reported having the initial symptoms of the disease.

The neuropsychological battery ${ }^{7}$ given to all patients in group 1 comprised immediate

Table 3 Mean (SD) values of factor scores between subgroups of group 1 with different initial symptoms and side effects when given levodopa

\begin{tabular}{llrr}
\hline Variable & Memory & \multicolumn{1}{l}{$\begin{array}{l}\text { Visuospatial } \\
\text { abilities }\end{array}$} & \multicolumn{1}{c}{$\begin{array}{l}\text { Psychomotor } \\
\text { speed }\end{array}$} \\
\hline Initial symptoms: & & & \\
Bilateral & $0.165(0.247)^{\star}$ & $0.472(1.192)$ & $-0.478(1.049)$ \\
Unilateral & $0.667(0.480)^{\star}$ & $0.138(0.982)$ & $0.156(0.986)$ \\
Right sided & $0.594(0.523)$ & $0.229(0.991)$ & $0.051(1.102)$ \\
Left sided & $0.806(0.363)$ & $-0.037(0.975)$ & $0.360(0.706)$ \\
Tremor & $0.638(0.542)$ & $0.213(0.996)$ & $0.200(1.016)$ \\
Other signs & $0.585(0.387)$ & $0.126(0.998)$ & $-0.107(0.956)$ \\
Side effects: & & & \\
Psychotic & $0.606(0.479)$ & $-0.258(1.054)$ & $-0.013(0.913)$ \\
No psychotic & $0.457(0.541)$ & $0.222(0.946)$ & $0.343(1.099)$ \\
Dyskinesia & $0.641(0.382)$ & $0.406(0.846)$ & $0.198(0.984)$ \\
Normokinesia & $0.457(0.541)$ & $0.222(0.946)$ & $0.343(1.099)$ \\
\hline$\star \mathrm{p}=0.005, t$ test. & & &
\end{tabular}

and delayed logical memory and immediate and delayed paired associates subtests of the Wechsler memory scale (WMS); trailmaking test (forms A and B); the block design, information, vocabulary, digit symbol, and digit span subtests of the Wechsler adult intelligence scale (WAIS); the Wepman visual discrimination and spatial orientation memory tests; maximum finger tapping rate on both sides, the Bender recall test, and a timed cancellation task. Depression was rated using the Zung self-rating depression scale. ${ }^{8}$

Patients in group 2 were given the mini mental state examination (MMSE) ${ }^{9}$ and a neuropsychological test battery including the selective reminding test ${ }^{10}$; Minnesota paper form board test ${ }^{11}$; the Wepman visual discrimination and Wepman spatial orientation memory tests ${ }^{12}$; and the similarities and digit span subtests of the WAIS-R. ${ }^{13}$ Depression was assessed by the geriatric depression scale. ${ }^{14}$

To assess the maximum speed of arm movements, patients pursued a moving spot of light on a cathode ray tube by covering it with a photocell held in their outstretched hand. The spot jumped to an unpredictable location each time the patient "caught" it. A pursuit score was computed as the number of contacts with the target in 50 seconds. This test, carried out for the left and right arm separately, was given on every visit to the clinic. The rates of change of motor speed on the right and left sides were estimated from the slopes obtained when the pursuit scores were regressed on time during a period when the patients were optimally medicated. Only those subjects with at least 10 serial assessments obtained over a period of at least two years were used for this analysis.

Data were analysed with the SYSTAT statistical package. ${ }^{15}$ Analyses included $t$ tests, principal components analysis, Pearson correlation, analysis of covariance, and logistic regression. Variables for which distributions were skewed were normalised by log transformation.

\section{Results}

GROUP 1

Complete neuropsychological data were available for 50 of 60 patients with Parkinson's disease in this group. A principal components analysis with varimax rotation was performed on these data, yielding three factors that explained $59 \cdot 6 \%$ of total variance (table 2 ). The first factor loaded primarily on measures of short term, long term, and remote memory; the second on visuospatial abilities; and the third on measures of psychomotor speed. None of the factors was significantly correlated with the Zung depression score.

Factor scores were constructed for all patients and distributions obtained. Scores for factor 1 (memory) were $\log$ transformed to normalise the distribution. The means of the three factor scores were compared between subgroups with different initial symptoms and profiles of side effects when first given levodopa (table 3). Patients whose initial 
Table 4 Analyses of covariance with memory as the dependent variable and initial symptoms as independent variables controlling for age and duration of parkinsonian symptoms in group 1

\begin{tabular}{lll}
\hline & \multicolumn{2}{l}{ Memory } \\
\cline { 2 - 3 } & $F$ & $p$ Value \\
\hline Analysis 1: & & \\
$\quad$ Bilateral v unilateral symptoms & 5.209 & 0.028 \\
Age & 0.777 & 0.383 \\
Duration of parkinsonian symptoms & 0.025 & 0.875 \\
Analysis 2: & & \\
$\quad$ Right sided v left sided symptoms & 2.672 & 0.111 \\
Age & 1.465 & 0.234 \\
$\quad$ Duration of parkinsonian symptoms & 0.029 & 0.866 \\
Analysis 3: & & \\
$\quad$ Tremor v other symptoms & 0.057 & 0.813 \\
Age & 0.586 & 0.448 \\
Duration of parkinsonian symptoms & 0.051 & 0.822 \\
\hline
\end{tabular}

symptoms were bilateral ( $v$ unilateral) had significantly greater memory impairment ( $t$ test, $p=0.005$ ), but did not differ significantly on visuospatial ability or psychomotor speed. To assess the role of the type of symptom, we divided patients into those whose initial symptom was tremor alone $(57 \cdot 4 \%) v$ those who had at least one other motor symptom at disease onset $(42 \cdot 5 \%)$. No significant differences were found for patients presenting with right $v$ left sided symptoms or for patients presenting with tremor $v$ those presenting with other motor signs. Patients presenting with psychotic side effects to levodopa did not differ in their cognitive performance from those in whom psychotic side effects were not present. Similarly, no differences were apparent between those presenting with dyskinesias and those without these side effects when levodopa was first given.

To determine the effect of the distribution and type of initial motor symptoms on memory performance, controlling for age and duration of parkinsonian symptoms, a series of analyses of covariance were performed (table 4). A significant effect was present for bilateral $v$ unilateral presentation of symptoms (analysis 1). Other characteristics of presentation, including the laterality of symptoms (right $v$ left) and the type of initial symptoms (tremor $v$ other symptoms) were not significantly associated with the degree of memory impairment (analyses 2 and 3). Furthermore, neither of the covariates (age and duration of

Table 5 Analyses of covariance with selective reminding test as the dependent variable and initial symptoms as independent variables controlling for age and duration of parkinsonian symptoms in group 2

\begin{tabular}{lll}
\hline & \multicolumn{2}{l}{ Selective reminding test } \\
\cline { 2 - 3 } Variable & $F$ & $p$ Value \\
\hline Analysis 1: & & \\
$\quad$ Bilateral v unilateral symptoms & 3.466 & 0.065 \\
Age & 14.551 & 0.000 \\
$\quad$ Duration of parkinsonian symptoms & 1.098 & 0.297 \\
Analysis 2: & & \\
$\quad$ Right sided v left sided symptoms & 0.040 & 0.843 \\
Age & 6.176 & 0.015 \\
$\quad$ Duration of parkinsonian symptoms & 0.494 & 0.484 \\
Analysis 3: & & \\
$\quad$ Tremor v other symptoms & 4.546 & 0.035 \\
Age & 10.640 & 0.001 \\
Duration of parkinsonian symptoms & 0.737 & 0.392 \\
\hline
\end{tabular}

parkinsonian symptoms) was significantly related to performance on the memory tests. Factors 2 and 3 (visuospatial and psychomotor speed) were assessed by similar analyses of covariance, but no significant associations were found with respect to the type or distribution of initial motor symptoms.

In a subset of 27 patients, data were available on the rate of decline of motor speed over time (slope of pursuit score $v$ time). The rate of decline of motor speed in the right arm was significantly correlated with the degree of memory impairment $(r=0.436, \mathrm{p}<0.05)$, as was the rate of decline of motor speed in the left arm $(r=-0.400, \mathrm{p}<0.05)$. To control for severity we separated out the pursuit scores obtained at the time of neuropsychological battery administration. This resulted in no change in the association between the rate of decline of right arm motor speed and memory $\left(r_{\text {partial }}=0.444, \mathrm{p}<0.05\right)$. The association between the rate of decline of left arm speed and memory, however, lost significance $\left(r_{\text {partial }}\right.$ $=0.343, \mathrm{NS}$ ).

\section{GROUP 2}

Patients in group 2 were divided into those with MMSE scores < 24 ( $n=36$, "cognitively impaired") and those with MMSE scores $>23$ ( $n=123$, "intact"). The cognitively impaired patients were significantly older (68.5 $v 65.8$ years, $t=1.95, \mathrm{p}=0.05)$, but did not differ in duration of parkinsonian symptoms (9.3 $v 9.3$ years, $t=0.99$, NS) or education $(12.2 v 11.3$ years, $t=1.41, \mathrm{p}=$ $0 \cdot 16)$. Depression scores also did not differ between these groups $(10.6 v 10.5, t=0.98$, NS).

Odds ratios (ORs) for cognitive impairment were estimated from logistic regression, controlling for age and duration of parkinsonian symptoms. The OR for cognitive impairment was not significant for the type of presenting symptoms (tremor only $v$ other symptoms OR $=1 \cdot 2,95 \%$ confidence interval $(95 \% \mathrm{CI})$ $0 \cdot 6-2 \cdot 5)$ or for initial symptoms involving the lower $v$ those restricted to the upper extremity $(\mathrm{OR}=1 \cdot 7,95 \%$ CI $0 \cdot 7-3 \cdot 8)$. The OR for bilateral $v$ unilateral presentation was significant $(\mathrm{OR}=2 \cdot 4,95 \% \mathrm{CI} 1 \cdot 0-5 \cdot 8)$ and that for right $v$ left sided unilateral presentation almost reached significance $(\mathrm{OR}=2 \cdot 7,95 \%$ CI 0.9-8.3).

A principal components analysis on the neuropsychological data was performed and yielded three factors that explained $71.9 \%$ of total variance. Because of missing data for some subtests, the variables with highest loadings in each factor were selected for study. They were the selective reminding test (logarithm of long term retrieval score) for factor 1 , Wepman visual discrimination test for factor 2 , and WAIS digit span for factor 3 .

Analyses of covariance were performed to identify significant differences between subgroups with different patterns of initial symptoms, controlling for age and duration of the disease (table 5). When the sum of long term retrieval on the selective reminding test was the dependent variable, patients presenting 
with tremor $v$ other symptoms were more likely to be impaired $(F=4.546, \mathrm{p}=0.035$; analysis 3) and there was a strong trend for those presenting with bilateral impairment to have greater deficits on this measure as well $(F=3.466, \mathrm{p}=0.065$; analysis 1$)$. No differences were apparent for right $v$ left sided disease presentation. In all of the analyses, there was a significant effect of age, with older patients showing greater impairment. Parallel analyses were performed with scores on the Wepman visual discrimination test and WAIS digit span as dependent variables, but no significant associations were found with presenting motor symptoms.

\section{Discussion}

The most consistent finding of this study was that bilateral ( $v$ unilateral) symptoms at disease onset were more often associated with memory impairment and low MMSE scores an average of nine years later. Reid et al ${ }^{6}$ reported that late onset patients with bilateral symptoms had significantly greater impairment on an auditory verbal learning test in comparison with those who only had unilateral symptoms. This finding was obtained, however, with simultaneous observations of motor and cognitive function after the disease was well established. Our results suggest that patients who present initially with bilateral motor symptoms may have a more aggressive disease course than patients who present with unilateral motor symptoms, including a greater risk of cognitive impairment several years after disease onset. The involvement of both sides of the brain early in the disease course could result in a reduced capacity to compensate for damage to areas of the brain involved in memory or cognition or may be a marker for more rapid progression of the disease. The second explanation is supported by the fact that patients presenting with bilateral symptoms also showed a higher rate of decline in pursuit score than did patients with unilateral symptoms at onset $(t=3.318, \mathrm{p}=$ $0.004)$.

In a subset of patients, we also found that a higher rate of decline of arm movement speed predicted increased memory impairment. Several investigators have noted associations between the severity of bradykinesia and cognitive impairment. Portin et al ${ }^{16}$ reported a significant correlation between the severity of bradykinesia and memory deficits. This finding was confirmed by Reid et al, ${ }^{6}$ who found that patients with more severe bradykinesia performed worse on tests assessing auditory verbal learning, verbal fluency, visuospatial skill, visual memory, and reaction time. Other investigators, including Mortimer et $a l^{5}$ and Iwasaki et $a l,{ }^{17}$ found the degree of bradykinesia to be correlated with the severity of impairment on visuospatial tasks. The present results are the first to show an association between the rate of progression of bradykinesia and the severity of memory impairment controlling for the severity of bradykinesia at the time of neuropsychological testing.
There was a trend for patients in group 2 with right ( $v$ left sided) onset to show greater impairment on the MMSE score. No associations with laterality of initial symptoms were evident in group 1 , however, and performance on the selective reminding test was unrelated to right ( $v$ left) sided presentation in group 2 . Simultaneous assessment of motor and cognitive deficits have shown various associations between motor symptom laterality and cognitive impairment. Spicer et $a l^{18}$ found that patients with unilateral right sided motor signs had greater impairment on serial digit learning, confrontation naming, and verbal associative fluency than patients with left sided disease. Blonder et al ${ }^{19}$ reported an association between right sided symptoms and verbal deficits and between left sided symptoms and visuospatial impairment. Huber et $a l^{20}$ and Oyebode et $a l,{ }^{21}$ however, found no differences in the pattern of neuropsychological deficits between patients with right sided symptoms and those with left sided symptoms. The present findings relate the side of presenting motor symptoms to cognitive impairment later in the disease course. Because most of our patients presenting with unilateral motor signs progressed to bilateral disease, comparisons with previous cross sectional studies where motor and cognitive disorders were assessed simultaneously are inappropriate. It is interesting to note, however, that the one measure showing a suggestive association with the laterality of presenting motor signs, the score on the MMSE, is highly dependent on language function, ${ }^{22}$ consistent with the left hemisphere (right sided motor symptom) involvement.

In one analysis, where the sum of long term retrieval of words on the selecting reminding test was used as the dependent variable, we found that patients presenting with tremor $v$ other motor symptoms were more likely to show impairment later in the disease course. This association was not present for any other dependent variable, including factor scores for memory performance in group 1 and the MMSE score in group 2 . It is possible that this association may have occurred by chance or may be related to the particular measure used.

Neither psychotic nor dyskinetic side effects when levodopa was first given predicted later impairment on neuropsychological tests. This is in agreement with the finding of Danielczyk and Fischer ${ }^{23}$ that there was no significant difference between the mean MMSE scores of non-demented parkinsonian patients with and without reversible psychosis.

Previous studies in which cohorts of parkinson patients were followed prospectively found that higher age and age of onset, ${ }^{24-26}$ severity of motor manifestations, ${ }^{2627}$ and the presence of depression ${ }^{26} 27$ were associated with an increased risk for incident dementia. Ebmeier et $a l^{28}$ reported that a history of smoking 3.5 years before assessment was associated with a diagnosis of prevalent dementia in Parkinson's disease, whereas 
Salganik and Korczyn ${ }^{29}$ found an association between hypertension and dementia in this illness. These findings may reflect the increased risk of cerebrovascular disease and stroke with smoking and hypertension, which could lower the threshold for symptoms of dementia.

In the present study, higher age was found to increase the risk of poor performance on the selective reminding test in group 2 , but to have no independent effect on neuropsychological performance in group 1 when duration of parkinsonian symptoms and presenting motor symptoms were controlled. Patients in group 2 with cognitive impairment assessed by low scores on the MMSE, however, were significantly older than those with high scores on this test. Because depression was not assessed before the neuropsychological testing, it was not possible to examine the interesting association between cognitive impairment and antecedent depression evident in two recent studies. ${ }^{27}{ }^{30}$ The finding that patients with more severe motor symptoms were more likely to become demented is consistent with the findings from the present study that presentation with bilateral symptoms and more rapid progression of bradykinesia are predictive of later cognitive impairment.

Because the patients in our study were clinically diagnosed cases, it is possible that some of them, and in particular those with pronounced cognitive impairment, might have other diseases. We were particularly careful, however, not to include in our analyses patients who were cognitively impaired at the onset of motor symptoms, making it less likely that another disease with parkinsonian features explained the associations.

This work was supported by the Swedish Medical Research Council, the Swedish Society of Medicine, Osterman's and Stohne's Foundations, the Wenner-Gren Foundation, and the Veterans' Affairs Medical Center.

1 Levin BE, Tomel R, Rey GJ. Cognitive impairment in Parkinson's disease. Neurol Clin 1992;10:471-85

2 Mortimer JA, ed. Special issue on Parkinson's disease. Alzheimer Dis Assoc Disord 1990;4:129-80.

3 Copper JA, Sagar HJ, Jordan N, Harvey NS, Sullivan EV. Cognitive impairment in early, untreated Parkinson's disease and its relationship to motor disability. Brain disease and its relation

4 Pillon B, Dubois B, Cusimano G, Bonnet A-M, Lhermitte F, Agid Y. Does cognitive impairment in Parkinson's disease result from non-dopaminergic lesions? $\mathcal{f}$ Neurol Neurosurg Psychiatry 1989;52:201-6.
5 Mortimer JA, Pirozzolo FJ, Hansch EC, Webster DD. Relationship of motor symptoms to intellectual deficits in Parkinson's disease. Neurology 1982;32:133-7.

6 Reid WGJ, Broe GA, Hely MA, et al. The neuropsychology of de novo patients with idiopathic Parkinson's disease: the effects of age of onset. Int $\mathcal{F}$ Neurosci 1989 ; 48:205-17.

7 Pirozzolo FJ, Hansch EC, Mortimer JA, Webster DD Dementia in Parkinson disease: a neuropsychological Dementia in Parkinson disease:

8 Zung WWK. A self rating depression scale. Arch Gen Psychiatry 1965;12:63-70.

9 Folstein MF, Folstein SE, McHugh PR. 'Mini-Mental State'. A practical method for grading the cognitive state of patients for the clinician. $\mathcal{F}$ Psychiatr Res 1975 12:189-98.

10 Buschke H, Altman Fuld PA. Evaluating storage, retention and retrieval in disordered memory and learning. Neurology 1974;24:1019-25.

11 Likert R, Quasha WH. The revised Minnesota paper form board test. manual. New York: Psychological Corporation, 1970.

12 Wepman JM, Turaids D. Spatial orientation memory test. Manual and directions. Palm Springs, test. Malifornia:Language Research Associates, Chicago: California:Lang

13 Wechsler D. WAIS-R manual. New York: Psychological Corporation. 1981 .

14 Yesavage JA, Brink TL, Rose RL, et al. Development and validation of a geriatric depression screening scale: a preiminary report. F. Psychiatr Res 1983;17:37-49.

15 Wilkinson, L. SYSTAT: the system for statistics. Evanston IL: SYSTAT, Inc, 1989

16 Portin RI, Koivusalo A, Laihinen AO, Rinne UK Cognitive deficits, motor disability and duration of illness in early Parkinson's disease. 7 Clin Exp Neuropsycho $1989 ; 11 ; 369$.

17 Iwasaki Y, Kinoshita M, Ikeda K, Takamiya K. Cognitive function in Parkinson's disease: in relation to motor symptoms. Int $\mathcal{f}$ Neurosci 1989;47:295-300.

18 Spicer KB, Roberts RJ, LeWitt PA. Neuropsychological performance in lateralized parkinsonism. Arch Neurol performance in

19 Blonder LX, Gur RE, Gur RC, Saykin AJ, Hurtig HI. Neuropsychological functioning in hemiparkinsonism. Brain Cogn 1989;9:244-57.

20 Huber SJ, Freidenberg DL, Shuttleworth EC, Paulson GW, Clapp LE. Neuropsychological similarities in lateralized parkinsonism. Cortex 1989;25:461-70.

21 Oyebode JR, Barker WA, Blessed G, Dick DJ, Britton PG Cognitive functioning in Parkinson's disease: in relation to prevalence of dementia and psychiatric diagnosis. $\mathrm{Br} f$ Psychiatry 1986;149:720-5.

22 Tombaugh TN, McIntyre NJ. The mini-mental state exame review, 7 Am Geriatr Soc 1992;40:922-35.

23 Danielczyk W, Fischer P. Psychiatric complications and shift of death age in Parkinson's disease. Adv Neurol 1990;53:405-10.

24 Biggins CA, Boyd JL, Harrop FM, et al. A controlled, longitudinal study of dementia in Parkinson's disease.

25 Mayeux R, Denaro J, Hemenegildo N, et al. A populationbased investigation of Parkinson's disease with and without dementia. Relationship to age and gender. Arch Neurol 1992;49:492-7.

26 Stern Y, Tang M, Marder TK, Mayeux R. Antecedent risk factors for incident dementia in Parkinson's disease. Neurology 1992;42(suppl 3):193-4

27 Marder K, Tang M-X, Cote L, Stern Y, Mayeux R. Risk actors for dementia in Parkinson's disease. Neurology 1993;43(suppl 2):A285-6.

28 Ebmeier KP, Calder SA, Crawford JR, Stewart L, Besson JAO, Mutch WJ. Clinical features predicting dementia in idiopathic Parkinson's disease: a follow-up study. Neurology 1990;40:1222-4.

29 Salganik I, Korczyn A. Risk factors for dementia in Parkinson's disease. Adv Neurol 1990;53:343-7.

30 Starkstein SE, Bolduc PL, Mayberg HS, Preziosi TJ Robinson RG. Cognitive impairments and depression in Parkinson's disease: a follow up study. $\mathcal{f}$ Neurol Neurosurg Psychiatry 1990;53:597-602. 